\title{
Special issue on intelligent tools and techniques for signals, machines and automation
}

\author{
Smriti Srivastava, Hasmat Malik* and Rajneesh Sharma \\ Division of Instrumentation and Control Engineering, Netaji Subhas Institute of Technology (NSIT) Delhi, \\ New Delhi, India
}

This special issue is a selected collection of papers submitted to the First International Conference on Signals, Machines and Automation (SIGMA-2018), February 23-25, 2018, New Delhi, India. These papers have been reviewed and accepted for presentation at the conference and for publication in the Journal of Intelligent \& Fuzzy Systems (JIFS). In this special issue there are 49 papers covering a wide range of topics in the area of intelligent systems and their application in various domains.

The International Conference SIGMA-2018 aims to provide a common platform to the researchers in related fields to explore and discuss various aspects of artificial intelligence applications and advances in soft computing techniques. The Conference will provide excellent opportunities for the presentation of interesting new research results and discussion about them, leading to knowledge transfer and the generation of new ideas.

Among the 49 papers in this special issue, there are twelve papers addressing the applications of intelligent tools and techniques in the operation and control of power systems. In [1], the Ant Colony Optimization (ACO) algorithm is used for mitigating contingencies by means of reconfiguration in an electrical distribution network. The results are validated on the IEEE 30-bus system and show that the algorithm is able to better reduce real power losses

\footnotetext{
${ }^{*}$ Corresponding author. Hasmat Malik, Netaji Subhas Institute of Technology (NSIT) Delhi, Dwarka Sector-3, New Delhi, 110078, India. Tel.: +91 11 25000209; E-mail: hasmat.malik@gmail.com.
}

during an overload contingency. In [2], a multi-stage hybrid artificial intelligence based solution is proposed for the unit commitment problem in a mixed generation system with energy storage, where the results show a significant amount of savings with the proposed scheme. In [3], Fuzzy Reinforcement Learning is used to solve the Economic Thermal Power Dispatch problem in a multiple fuel system. The results indicate that the proposed algorithm emerges as a superior and alternative technique for the solution of the problem. In [4], an interline power flow controller (IPFC) along with a two degree-offreedom proportional integral derivative (TDOFPID) controller is used for enhancing the performance of automatic generation control (AGC) with the help of evolutionary algorithms. In [5], a genetic algorithm (GA) based approach is taken to optimize the performance of load frequency control in a deregulated scenario. The results show a significant improvement in performance of the GA optimized controller when compared to a traditional PID controller. In [6], an adaptive optimal control approach for AGC is proposed for a deregulated power system. In [7], a GA based optimal power flow (OPF) is used for the optimal procurement of energy as well as operating reserves. The results were validated on IEEE 30-bus system. In [8], the controller for an Automatic Voltage Regulator (AVR) is optimized using a multiobjective Non Dominated Sorting Genetic Algorithm II (NDSGA-II). The paper demonstrated the superiority of the algorithm in optimizing the controller when compared to several other evolutionary algorithms such as Artificial Bee Colony (ABC), Particle 
Swarm Optimization (PSO), Differential Evolution Algorithm (DEA) etc. In [9], a Multi Class Support Vector Machine (MC-SVM) was used for fault detection and classification in a multi-terminal HVDC transmission system. The classifier is demonstrated to be capable of accurately classifying LG faults with an overall accuracy over 99\%. In [10], a PSO based scheme is used for optimally tuning a Unified Power Flow Controller (UPFC) on a standard IEEE 30-bus system. In [11], a Quantum Particle Swarm Optimization technique is employed for solving the expansion planning problem in a 72-bus practical distribution network. The performance is compared to the standard PSO algorithm to illustrate the better performance of the proposed algorithm. In [12], an Adaptive Neuro Fuzzy Inference System (ANFIS) is used to enhance the performance of UPFC. The performance is compared to both conventional control schemes as well as a fuzzy controller.

There are seven papers that involve the use of intelligent techniques in the field of renewable energy. In [13], k-Nearest Neighbor (kNN) as well as Artificial Neural Network (ANN) is used to perform a deterministic and probabilistic wind speed forecasting methodology. The results show that while the Multi-Layer Perceptron (MLP) based ANN gives a forecasting accuracy of around 94\%, the kNN algorithm is able to improve this to over $99 \%$. In [14], a simultaneous optimization technique is proposed for a renewable energy based pumped storage scheme for the ancillary services market for a deregulated power sector. In [15], a multi-objective Glowworm Swarm Optimization technique is used for solving the optimal scheduling problem in a Thermal-Wind Power system. In [16], load frequency control is performed using a fractional order controller optimized using PSO for a solar-diesel based isolated power system. In [17], a BBBC based frequency controller is proposed for a hybrid solar thermal-wind power system. In [18], a mixed GA and optimal power flow (OPF) based model is used for determining the best location and rating for wind power generation in a competitive electricity market. In [19], various optimization techniques are used with a maximum power point tracking (MPPT) based solar battery charger system with a single current sensor for use in electric vehicles.

There are five papers in which various applications in the domain of healthcare are discussed where intelligent techniques have been employed. In [20], a layer recurrent neural network (LRNN) based user activity classifier is proposed which uses the sensors in a smartphone to determine the movements and activities of a person. The performance of the proposed classifier is shown to be significantly better than both a MLP based classifier and a Naive Bayes classifier. In [21], various intelligent techniques, such as KNN, linear discriminant analysis (LDA), quadratic discriminant analysis (QDA) and medium tree classifier, are used for the classification and feature extraction in surface electromyography (sEMG) signals. In [22], a neural reinforcement learning based classifier is used for identifying elbow, finger and hand movements. The classifier is employed in the identification of the elbow movement angle with an accuracy of $97.5 \%$, for the identification of finger movements when typing keys with an accuracy of $98.7 \%$ and for hand movements with an accuracy of $97.6 \%$. In [23], a SVM based classifier is used for translating magneto-encephalography (MEG) signals to the corresponding wrist movement and the results obtained have been compared to various other techniques employed for the same. In [24], neuro-fuzzy techniques have been employed in conjunction with failure modes and effects analysis (FMEA) to calculate the risk priority numbers (RPN) with the goal of improving the quality of service at hospitals.

There are five papers in the special issue where intelligent tools and techniques are applied to the operation of electric motors and the control of their drives. In [25], wavelet packet transform (WPT), principal component analysis (PCA) and dendogram support vector machine (DSVM) are used for the bearing fault classification of induction motors. In [26], a PI and Fuzzy based technique is used to improve the efficiency of vector control of an induction motor drive system. In [27], the performance of a boost converter with a fuzzy controller and active power factor correction is evaluated. In [28], sensorless field oriented control based drive for permanent magnet synchronous motors (PMSM) is implemented using various techniques such as ANN, model reference adaptive system (MRAS) and ANFIS. In [29], the performance of a fuzzy logic regulator (FLR) for a five phase permanent magnet synchronous machine (FPSM) is studied and compared with that of a traditional Integral Proportional (IP) regulator and better performance of the FLR is established in terms of the faster dynamics of the machine.

Various improved intelligent tools and techniques have been proposed in five papers. In [30], a new weight initialization method for feed forward artificial neural networks (FFANN) is proposed. 
Experiments performed on multiple datasets demonstrate the efficacy of the proposed scheme over the random weight initialization that is traditionally used with neural networks. In [31], a nature-inspired metaheuristic algorithm - moth flame optimization - is used for the generation of test sequences in state based testing. In [32], the authors discuss intuitionistic fuzzy parameterized fuzzy soft set (IFP-FS set) and its application in decision making problems. In [33], the authors introduce Interval Valued q-Rung Orthopair Fuzzy Sets (IVqROFSs) and present some of its important operations such as negation, union and intersection. Comparison and contrast is drawn with Intuitionistic Fuzzy Sets (IFS) and Pythagorean Fuzzy Sets (PFS). In [34], a novel squared pinball loss twin support vector regression model (SPTSVR) is proposed. The proposed model solves a pair of strongly convex minimization problems having unique solutions which are obtained by simple functional iterative method and a comparison with support vector regression (SVR), least squares support vector regression (LS-SVR) and twin support vector regression (TSVR) methods illustrate the effectiveness and a usefulness of the proposed scheme.

The application of intelligent techniques to image processing is a fast growing field of study. There are four papers from this domain. In [35], a technique for detection of copy-move forgery in images is presented which is capable of better detection of forged images with lesser number of features and computing power. The effectiveness of the technique as an image forensic tool is also demonstrated. In [36], a new Spatial Intuitionistic Fuzzy C-means (S-IFCM) technique is presented which uses Cityblock distance to compute the rank between two pixels. Experiments were performed on various noisy images including MRI brain image, to assess the performance of the proposed algorithm and comparisons were done with existing hard, fuzzy and intuitionistic methods to demonstrate the effectiveness of the proposed method. In [37], a technique for illumination normalization in images using homomorphic filtering and reflectance ratio is discussed which is capable of improving the performance of face recognition techniques under diverse illumination conditions. In [38], a real time novel algorithm to reckon number of eye blinks in a video sequence using eye facet correlation (EFC) is proposed. The proposed algorithms is classified with existing classifiers like SVM, QSVM, $\mathrm{KNN}$ and DTREE, and show an increase in accuracy to $99.8 \%$, validates the effectiveness and correctness of the algorithm.
There are 3 papers covering the use of fuzzy techniques in the domain of robotics. In [39], a fractional order fuzzy proportional derivative with fractional order integrator (FOFPD+FOI) controller is used for a three link robotic manipulator system and its performance is compared to that of PID, FOPID and IOFPD+IOI controllers. In [40], a robust fractional order fuzzy sliding mode PID controller (FOFSMCPID) is proposed for a two link robotic manipulator system and its performance is investigated in comparison with other control schemes for the robotic manipulator system. In [41], the performance of a neuro-fuzzy control scheme for a 6-degree of freedom (6-DoF) robotic arm is evaluated and compared to traditional PID controllers. The results showing the improvement in performance of the neuro-fuzzy control scheme is validated using experiments performed on a hardware test bench.

There are two papers covering the application of intelligent techniques in control systems. In [42], Grey Wolf Optimization (GWO) technique is employed in the control of a doha model reverse osmosis water treatment plant. The performance of the GWO-PID controller is compared with that of PID controller optimized using other meta-heuristic algorithms such as ABC and ACO. In [43], techniques of applying evolutionary algorithms for the modeling of fractional order chaotic systems are evaluated. The modeling of fractional order chaotic systems such as Financial System, Chen System, Lorenz's system and 3 Cell Net systems are considered and are modeled with the help of ABC and ACO algorithms.

The remaining papers of the special issue cover the application of intelligent techniques in a variety of different domains. In [44], a novel feature extraction technique for palmprint recognition is proposed using Oriented Gabor Gradients (OGG). The proposed technique is evaluated on multiple palmprint datasets and is compared to Histogram of Oriented Gradients (HOG), Gabor Transform, Gaussian Membership based Features (GMF), Absolute Average Deviation (AAD) and Mean Features to illustrate the superiority of the proposed OGG method. In [45], various supervised and unsupervised learning techniques are used to perform market segmentation for use in ecommerce applications. Self-Organized Map (SOM), SVM and KNN techniques are used to perform market segmentation using primary survey data collected from the users of e-commerce markets. Evolutionary algorithms are used to further improve the performance of these market segmentation techniques. In [46], an efficient multistage bandwidth allocation 
technique is proposed for the migration of virtual machines (VMs) in a cloud computing environment. In [47], a public key cryptography based user authentication scheme is discussed where the requirement of a smart card is eliminated. In [48], Empirical Mode Decomposition (EMD) and ANN based techniques are used for performing fault diagnosis in rolling bearings in a mechanical system. Various types of ANNs, such as MLP, Radial Basis Functions (RBF), Probabilistic Neural Network (PNN), Generalized Regression Neural Network (GRNN), Linear Vector Quantization (LVQ), are used for the fault classification.

Finally, in [49] the authors present an application of intelligence technique in civil engineering domain to analyze the structural health condition monitoring by using Gene Expression Programming (GEP). In this paper, authors have predicted the high performance concrete (HPC) compressive strength (HPCCS) at highly complex behavior.

In conclusion, this special issue would not have been possible without the help of many people. As guest editors, we would like to take this opportunity to thank the authors for their contributions and the reviewers for their invaluable comments and timely responses. We would like to thank NSIT for providing necessary resources \& platform and the division of instrumentation and control for their great management to give our delegates such a unforgettable experience of event. We would like to thank the UG, PG and Ph.D students for their tremendous work. We also would like to thank the JIFS Editor-in-Chief and staff for their support during the preparation and production of this special issue.

\section{References}

[1] M. Jamil and A. Sharma, Reconfiguration of Electrical Distribution system using ACO methodology, Journal of Intelligent \& Fuzzy Systems (JIFS) 35 (2018), 4901-4908.

[2] S. Tiwari, B. Dwivedi and M.P. Dave, A multi-stage hybrid artificial intelligence based optimal solution for energy storage integrated mixed generation unit commitment problem, Journal of Intelligent \& Fuzzy Systems (JIFS) 35 (2018), 4909-4919.

[3] N.K. Navin, R. Sharma and H. Malik, Solving nonconvex economic thermal power dispatch problem with multiple fuel system and valve point loading effect using Fuzzy Reinforcement Learning, Journal of Intelligent \& Fuzzy Systems (JIFS) 35 (2018), 4921-4931.

[4] D.K. Mishra, T.K. Panigrahi and P.K. Ray, Asit mohanty, performance enhancement of AGC under open market scenario using TDOFPID and IPFC controller, Journal of Intelligent \& Fuzzy Systems (JIFS) 35 (2018), 4933-4943.

[5] A. Singh, N. Kumar, B.P. Joshi and B.K. Singh, Load frequency control with time delay in restructured environment,
Journal of Intelligent \& Fuzzy Systems (JIFS) 35 (2018), 4945-4951.

[6] A. Singh, N.K.B. Prasad Joshi and K.S. Vaisla, AGC using adaptive optimal control approach in restructured power system, Journal of Intelligent \& Fuzzy Systems (JIFS) 35 (2018), 4953-4962.

[7] A. Banshwar, N.K. Sharma, B.B. Sharma, Y.R. Sood and R. Shrivastava, Mixed GA-OPF based optimal procurement of energy and operating reserve in deregulated environment, Journal of Intelligent \& Fuzzy Systems (JIFS) 35 (2018), 4963-4969.

[8] N.K. Yegireddy, S. Panda, T. Papinaidu and K. Praveen Kumar Yadav, Multi-objective non dominated sorting genetic algorithm-II optimized PID controller for automatic voltage regulator systems, Journal of Intelligent \& Fuzzy Systems (JIFS) 35 (2018), 4971-4975.

[9] M. Asim, A. Riyaz, S. Tiwari and A. Verma, Performance evaluation of fuzzy controller for boost converter with active PFC, Journal of Intelligent \& Fuzzy Systems (JIFS) 35 (2018), 5169-5175.

[10] S.P. Jaiswal and V. Shrivastava, A PSO based search for optimal tuning and fixing of UPFC to improve usefulness of distribution system, Journal of Intelligent \& Fuzzy Systems (JIFS) 35 (2018), 4987-4995.

[11] R.K. Malee, A.S. Chundawat, N. Maliwar and A.K. Sharma, DG integrated distribution system expansion planning with uncertainties, Journal of Intelligent \& Fuzzy Systems (JIFS) 35 (2018), 4997-5006.

[12] R.K. Chauhan, M. Hasan and J.P. Pandey, Intelligent control model to enhance the performance of unified power quality conditioner, Journal of Intelligent \& Fuzzy Systems (JIFS) 35 (2018), 5007-5020.

[13] A. Azeem and N. Fatema, $k$-NN and ANN based deterministic and probabilistic wind speed forecasting intelligent approach, Journal of Intelligent \& Fuzzy Systems (JIFS) 35 (2018), 5021-5031.

[14] S. Roy, A. Banshwar, N.K. Sharma and Y.R. Sood, Simultaneous optimization of renewable energy based pumped storage scheme in energy and ancillary services market under deregulated power sector, Journal of Intelligent \& Fuzzy Systems (JIFS) 35 (2018), 5033-5043.

[15] S.R. Salkuti and Y.H. Lho, Multi-objective glowworm swarm optimization for solving the optimal scheduling of thermal-wind power system, Journal of Intelligent \& Fuzzy Systems (JIFS) 35 (2018), 5045-5054.

[16] T. Mahto, H. Malik and M. Saad Bin Arif, Load frequency control of a solar-diesel based isolated hybrid power system by fractional order control using partial swarm optimization, Journal of Intelligent \& Fuzzy Systems (JIFS) 35 (2018), 5055-5061.

[17] B.K.S.N. Kumar, A. Singh and B. Prasad Joshi, BBBC based frequency controller for hybrid power system, Journal of Intelligent \& Fuzzy Systems (JIFS) 35 (2018), 5063-5070.

[18] N.K. Sharma, A. Banshwa, B.B. Sharma, Y.R. Sood and R. Shrivastava, Mixed GA-OPF based prioritized optimal location and rating of wind power generation in deregulated electricity market, Journal of Intelligent \& Fuzzy Systems (JIFS) 35 (2018), 5071-5075.

[19] A.Q. Ansari, M. Hasan and N. Islam, Novel optimization technique to charge E-rickshaw battery using single sensor based MPPT of SPV module, Journal of Intelligent \& Fuzzy Systems (JIFS) 35 (2018), 5077-5084.

[20] H. Jain and N. Fatema, Layer recurrent neural network based intelligent user activity classification model using 
smartphone, Journal of Intelligent \& Fuzzy Systems (JIFS) 35 (2018), 5085-5097.

[21] Y. Narayan, L. Mathew and S. Chatterji, sEMG signal classification with novel feature extraction using different machine learning approaches, Journal of Intelligent \& Fuzzy Systems (JIFS) 35 (2018), 5099-5109.

[22] A. Kukker and R. Sharma, Neural reinforcement learning classifier for elbow, finger and hand movements, Journal of Intelligent \& Fuzzy Systems (JIFS) 35 (2018), 5111-5121.

[23] A. Shahid, M. Wahab, N. Rafiuddin, M. Saad Bin Arif and H. Malik, Decrypting wrist movement from MEG signal using SVM classifier, Journal of Intelligent \& Fuzzy Systems (JIFS) 35 (2018), 5123-5130.

[24] N. Fatema, Application of neuro-fuzzy scheme to improve purchasing process in a hospital, Journal of Intelligent \& Fuzzy Systems (JIFS) 35 (2018), 5131-5146.

[25] S. Agrawal, V.K. Giri and A.N. Tiwari, Induction motor bearing fault classification using WPT, PCA and DSVM, Journal of Intelligent \& Fuzzy Systems (JIFS) 35 (2018), 5147-5158.

[26] M.S. Rajinder and M. Singh, Efficiency optimization of pi and fuzzy controller based induction motor drive, Journal of Intelligent \& Fuzzy Systems (JIFS) 35 (2018), 5159-5167.

[27] M. Asim, A. Riyaz, S. Tiwari and A. Verma, Performance evaluation of fuzzy controller for boost converter with active PFC, Journal of Intelligent \& Fuzzy Systems (JIFS) 35 (2018), 5169-5175.

[28] Md. Tabrez, F.I. Bakhsh, M. Hassan, K. Shamkanth and S. Al-Ghnimi, A comparative simulation study of different sensorless permanent magnet synchronous motor drives using neural network and fuzzy logic, Journal of Intelligent \& Fuzzy Systems (JIFS) 35 (2018), 5177-5184.

[29] M.A. Al-Hitmi, H. Kesraoui, K. Rahman and A. Iqbal, Comparative study of classical and fuzzy-regulator in five phase synchronous machine control with open phase, Journal of Intelligent \& Fuzzy Systems (JIFS) 35 (2018), 5185-5191.

[30] M.P.S. Bhatia, Veenu and P. Chandra, A new weight initialization method for sigmoidal FFANN, Journal of Intelligent \& Fuzzy Systems (JIFS) 35 (2018), 5193-5201.

[31] R. Sharma and A. Saha, Optimal test sequence generation in state based testing using moth flame optimization algorithm, Journal of Intelligent \& Fuzzy Systems (JIFS) 35 (2018), 5203-5215.

[32] B. Prasad Joshi, A. Kumar, A. Singh, P.K. Bhatt and B.K. Bharti, Intuitionistic fuzzy parameterized fuzzy soft set theory and its application, Journal of Intelligent \& Fuzzy Systems (JIFS) 35 (2018), 5217- 5223.

[33] B. Prasad Joshi, A. Singh, P.K. Bhatt and K.S. Vaisla, Interval valued $q$-rung orthopair fuzzy sets and their properties, Journal of Intelligent \& Fuzzy Systems (JIFS) 35 (2018), 5225-5230.

[34] P. Anagha, S. Balasundaram and Y. Meena, On robust twin support vector regression in primal using squared pinball loss, Journal of Intelligent \& Fuzzy Systems (JIFS) 35 (2018), 5231-5239.

[35] C.S. Prakash, S. Maheshkar and V. Maheshkar, Detection of copy-move image forgery with efficient block representation and discrete cosine transform, Journal of Intelligent \& Fuzzy Systems (JIFS) 35 (2018), 5241-5253.

[36] J. Arora and M. Tushir, Robust spatial intuitionistic fuzzy C-means with city-block distance clustering for image segmentation, Journal of Intelligent \& Fuzzy Systems (JIFS) 35 (2018), 5255-5264.

[37] J. Yadav, N. Rajpal and R. Mehta, A new illumination normalization framework via Homomorphic filtering and Reflectance ratio in DWT domain for face recognition, Journal of Intelligent \& Fuzzy Systems (JIFS) 35 (2018), 5265-5277.

[38] P.S. Lamba and D. Virmani, Reckoning number of eye blinks using eye facet correlation for exigency detection, Journal of Intelligent \& Fuzzy Systems (JIFS) 35 (2018), 5279-5286.

[39] J. Kumar, V. Kumar and K.P.S. Rana, A fractional order fuzzy PD+I controller for three-link electrically driven rigid robotic manipulator system, Journal of Intelligent \& Fuzzy Systems (JIFS) 35 (2018), 5287-5299.

[40] J. Kumar, V. Kumar and K.P.S. Rana, Design of robust fractional order fuzzy sliding mode PID controller for two-link robotic manipulator system, Journal of Intelligent \& Fuzzy Systems (JIFS) 35 (2018), 5301-5315.

[41] S. Srivastava, P.G. Aiswarya, M. Gupta, N. Prasannakumar, A. Rudola, A. Mallick and S. Srivastava, A comparative study of control of 6-DoF robotic arm using PID controller and ANFIS, Journal of Intelligent \& Fuzzy Systems (JIFS) 35 (2018), 5317-5327.

[42] N.S. Rathore, V.P. Singh and B. Kumar, Controller design for doha water treatment plant using grey wolf optimization, Journal of Intelligent \& Fuzzy Systems (JIFS) 35 (2018), 5329-5336.

[43] S. Gupta, V. Upadhyaya and A. Singh, Pragya varshney and smriti srivastava, modeling of fractional order chaotic systems using artificial bee colony optimization and ant colony optimization, Journal of Intelligent \& Fuzzy Systems (JIFS) 35 (2018), 5337-5344.

[44] Gopal, S. Srivastava,V. Kalra and A. Joshi, Palmprint recognition using novel oriented gabor gradients, Journal of Intelligent \& Fuzzy Systems (JIFS) 35 (2018), 5345-5351.

[45] R. Tiwari, M.K. Saxena, P. Mehendiratta, K. Vatsa, S. Srivastava and R. Gera, Market segmentation using supervised and unsupervised learning techniques for E -commerce applications, Journal of Intelligent \& Fuzzy Systems (JIFS) 35 (2018), 5353-5363.

[46] A. Bhardwaj and C.R. Krishna, Efficient multistage bandwidth allocation technique for virtual machine migration in cloud computing, Journal of Intelligent \& Fuzzy Systems (JIFS) 35 (2018), 5365-5378.

[47] D. Kumar, S. Chand and B. Kumar, A PKC-based user authentication scheme without smart card, Journal of Intelligent \& Fuzzy Systems (JIFS) 35 (2018), 5379-5390.

[48] H. Malik, A. Yadav and A.K. Shah, EMD and ANN based intelligent model for bearing fault diagnosis, Journal of Intelligent \& Fuzzy Systems (JIFS) 35 (2018), 5391-5402.

[49] S. Saad and H. Malik, Gene expression programming (GEP) based intelligent model for high performance concrete comprehensive strength analysis, Journal of Intelligent \& Fuzzy Systems (JIFS) 35 (2018), 5403-5418. 